Forum 2017 · 32:372

https://doi.org/10.1007/s12312-017-0339-2

Online publiziert: 4. Oktober 2017

๑) Springer Medizin Verlag GmbH 2017

Peter Albers

Präsident der Deutschen Krebsgesellschaft e.V.

\title{
„Qualität in der Krebsversorgung hat viele Aspekte."
}

gelassen werden. Und es gehört zu unserem Verständnis von Qualität in der ärztlichen Versorgung, im Gespräch mit den Patienten (und der Öffentlichkeit) auf bestehende Evidenzlücken aufmerksam zu machen.

Qualität in der Krebsversorgung hat viele Aspekte, nicht nur im nationalen Kontext. Auch in Europa bewegt sich einiges in diesem Bereich. Die DKG beteiligt sich seit Jahren aktiv an verschiedenen EU-Initiativen, die auf ein gemeinsames Verständnis von Qualität und Qualitätsmanagement der Krebsversorgung abzielen. Dazu gehören die CanCon Joint Action on Cancer Control, die 2017 mit der Fertigstellung des CanCon Guides endete, sowie die European Commission Initiative on Breast Cancer (ECIBC) und jetzt ganz aktuell die Joint Action - Innovative Partnership on Action Against Cancer. Bei unserer Arbeit in diesen Gremien kommt uns zugute, dass wir, verglichen mit anderen europäischen Ländern, die längste Erfahrung mit der Zertifizierung von Krebszentren sowie die größte Anzahl an zertifizierten Zentren aufweisen und außerdem mit tumorspezifischen Zertifizierungen aufwarten können.

Da liegt die Frage nahe, ob nicht auch andere Länder oder Gesundheitssysteme ein Interesse an der Adaptation dieses Zertifizierungssystems haben. Mit der Etablierung zertifizierter Zentren im deutschsprachigen Ausland, also der Schweiz, Österreich und Südtirol, wurde der erfolgreiche Anfang einer Ausweitung der Zertifizierung gemacht. Seit 2016 besteht die Möglichkeit, sich als European Cancer Centre zertifizieren zu lassen. In- zwischen liegen weitere Anfragen vor, unter anderem aus Luxemburg und Italien. Auch die European Association of Urology hat Interesse an einem Erfahrungsaustausch.

Diese Ausweitung ist sicher mit Herausforderungen verbunden. Welche Rolle spielt die jeweilige Landessprache für das Qualitätsmanagement? Wie geht man mit länderspezifischen Unterschieden in der Datenerhebung um? Was, wenn bestimmte Therapien in anderen Gesundheitssystemen nur eingeschränkt zugänglich sind? Bei einer Adaption wird man sich zunächst beschränken müssen: auf die drei häufigsten Tumorarten und auf ein "Core Module“, eine einheitliche Grundlage von Qualitätsanforderungen, die für möglichst viele Länder gelten kann. Das ist sicher keine einfache Aufgabe, trotzdem werden wir sie proaktiv angehen. Denn erstens können wir in punkto Qualitätsmanagement eine Menge dabei lernen. Und zweitens: Wenn wir nicht aktiv werden, dann ergreifen andere die Gelegenheit - mit der Gefahr, dass dann möglicherweise kommerzielle Interessen das Feld bestimmen.

Ihr

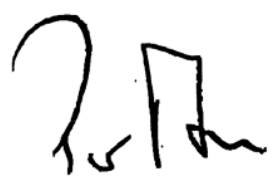

Peter Albers

Präsident der Deutschen Krebsgesellschaft 